\title{
Evoked potential in the visual cortex as modified by enucleation of one eye in the albino rat
}

\author{
MAKOTO SAKAI, FUMIO YAGI, and YUKINOBU IKEDA \\ Saga Medical School, Saga, Japan
}

\begin{abstract}
Evoked potential was recorded from the visual cortex to stimulation of the uncrossed optic nerve in the rat with one eye removed at birth. In the control adult rat, whose eye was enucleated immediately before the recording, the potential consisted of one small cortical wave, and it was recordable only from a limited portion of the visual cortex. In contrast, in the rat whose eye was enucleated at birth, the potential consisted of two cortical waves which were both potentiated and recordable from a large portion of the visual cortex. The first wave in this rat corresponded to the wave recorded in the control rat and was believed to be mediated by the geniculostriate projection. The second wave specifically related to the enucleation time mentioned above was assumed to be mediated by the superior colliculus (SC), the lateral posterior nucleus of the thalamus (LPT) projection. A possible neuronal reorganization underlying the appearance of the second wave was discussed.
\end{abstract}

It has been known that removal of one eye in the rat and hamster at birth or in early postnatal days produces an increase in the number of uncrossed optic nerve terminals in the lateral geniculate body (LBG) (Lund, Cunningham, \& Lund, 1973) and in the superior colliculus (SC) (Finlay, Wilson, \& Schneider, 1979; Frost \& Schneider, 1979; Lund et al., 1973; Lund \& Lund, 1973). Among many interesting questions to be asked concerning this phenomenon, an answer to the question of the functional capability of such terminals would be most interesting. Recent electrophysiological studies have demonstrated that they are functional both in LGB (Sakai \& Yagi, 1981) and in SC (Cunningham \& Speas, 1975; Finlay et al ., 1979; Rhoades, 1980; Takeuchi, Fukuda, Hara, \& Hsiao, 1982; Thompson, 1979). A question that follows concerns the visual cortex, which receives its main afferents from LGB and how it is functionally affected by such monocular enucleation. Sakai and Yagi (1981) have shown that if one eye is enucleated within 30 postnatal days in the rat, stimulation of the remaining optic nerve evokes a potential in the ipsilateral LGB, a postsynaptic component of which is larger and more

This investigation was supported in part by a grant-in-aid for scientific research (No. 56510065) from the Ministry of Education, Science, and Culture of Japan. The second author's present address is Vision Research Laboratory, Miyagi University of Education, Sendai 980, Japan. The third author's permanent address is Department of Psychology, Saga Women's Junior College, Saga 840, Japan. Requests for reprints should be directed to: Makoto Sakai, Neuropsychology Laboratory, Saga Medical School, 421-6 Nabeshima, Nabeshima-Machi, Saga 840-01, Japan. widely distributed than in normal rats. These findings suggest that the corresponding functional changes should be observed in the visual cortex, too. The present study was undertaken to examine this hypothesis; a potential evoked by stimulation of the optic nerve was mapped in the visual cortex using rats with one eye enucleated at birth and intact rats of more than 3 months of age. It should be noted here that the present study was specifically concerned with a functional reorganization of the visual cortex directly receiving either a crossed or uncrossed optic projection.

\section{METHOD}

\section{Subjects}

Adult male and female albino rats of the Wistar strain with either both eyes intact or the right eye removed at birth served as subjects. Their body weight ranged from 270 to $400 \mathrm{~g}$.

\section{Procedures}

The rats were anesthetized with urethane $(150 \mathrm{mg} / 100 \mathrm{~g}$, ip), and the heads were fixed in a stereotaxic apparatus in such a way as to allow the atlas of the rat brain provided by König and Klippel (1963) to be used. In all the animals studied, a length of 5-6 mm of the left optic nerve was exposed and hooked up to two U-shaped stainless steel electrodes ( $200 \mu \mathrm{m}$ in diameter) that were used for stimulation of the nerve. One electrode was positioned on the optic disk and the other on the nerve $2 \mathrm{~mm}$ closer to LGB from the optic disk. Throughout the experiment, rectal temperature was maintained at $36^{\circ}-38^{\circ} \mathrm{C}$ with a heating pad.

A large craniotomy was made on the dorsal portion of the occipital bone on either the right or left hemisphere, using a dental drill. Great care was taken to leave the dura intact; the exposed cortex was carefully prevented from drying by forming a thin layer of agar on it. Also, in order to record potentials in the visual cortex evoked by impulses exclusively mediated by either a crossed or uncrossed optic pathway, the optic tract either ipsilateral or contra- 


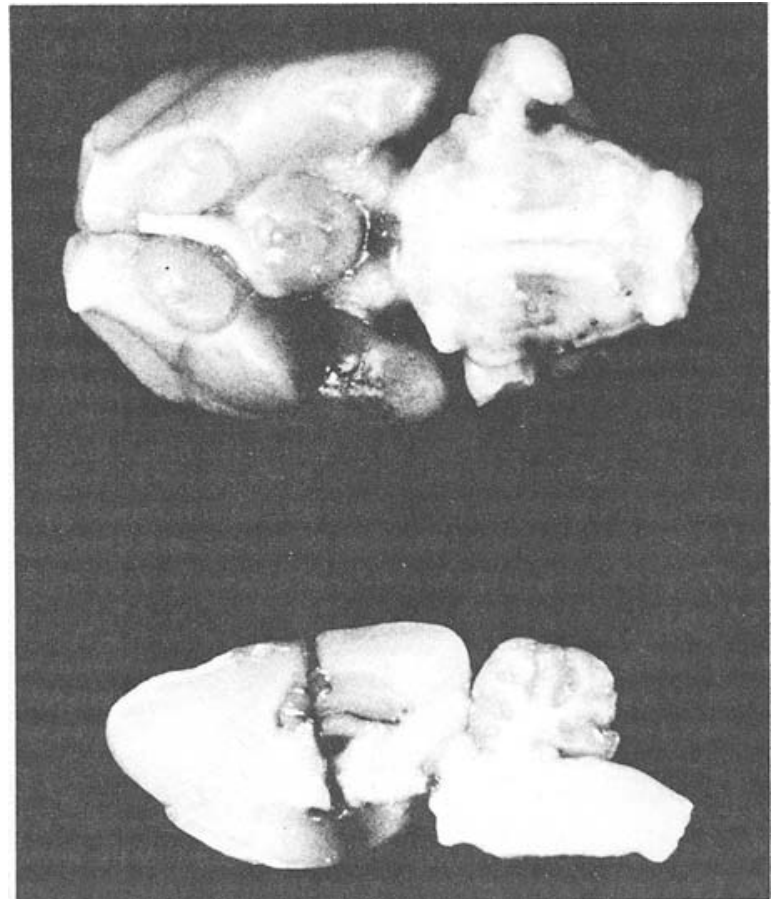

Figure 1. Ventral and saggital ( $3 \mathrm{~mm}$ lateral to the midline) views of spatula-cut traces in the OEB rat's brain.

lateral to the site of optic nerve stimulation was severed. The severance was made by stereotaxically lowering a smooth spatula ( $4 \mathrm{~mm}$ wide) from the cortex in the area $4 \mathrm{~mm}$ posterior to the bregma and 1.5 to $5.5 \mathrm{~mm}$ lateral to the midline until it reached the floor of the skull. Although the procedure was rather crude, it effectively blocked the impulses going up to visual centers with no observable influence on electrical activity in the opposite hemisphere.

A cortical evoked potential (CEP) was recorded by using bipolar concentric stainless steel electrodes (inner electrode, $100 \mu \mathrm{m}$; outer electrode, $500 \mu \mathrm{m}$ in diameter), which were insulated except for a cross section of the tips, with the inner tip $1 \mathrm{~mm}$ longer than the outer one. The electrodes were so positioned on the cortex that the outer tip could rest on the surface. CEP was recorded in the area $0 \mathrm{~mm}$ (A0) to $5 \mathrm{~mm}$ (A5) anterior to the interaural line (A0), and $2 \mathrm{~mm}$ (L2) to $5 \mathrm{~mm}$ (L5) lateral from the midline with a $1-\mathrm{mm}$ interval for both directions where $A$ and $L$ represent the anteroposterior and mediolateral coordinates based on the atlas provided by König and Klippel (1963). The recording was made medial to lateral in the order of $A$ at $0,1,2,3,4$, and $5 \mathrm{~mm}$, bringing the total number of the recording sites to 24 . Before a systematic recording of CEP was undertaken, a threshold intensity of the optic nerve stimulation for evoking a slight sign of CEP was determined by recording CEP at the site of A1 and L3. Unless otherwise mentioned, CEPs recorded at all 24 sites were obtained by using an intensity of stimulation five times this threshold value. CEPs were recorded at least $3 \mathrm{~min}$ after the electrode placement at each recording site. It should also be mentioned that the recording of CEPs was initiated 2.5 to $3 \mathrm{~h}$ after injection of urethane. The present study was primarily concerned with the analysis of waves or components of CEP which were definitely cortical in origin. Therefore, an attempt was made to record CEPs in some intact rats and in rats monocularly enucleated at birth in the period when the exposed cortex for recording CEPs was depressed by irrigation of physiological saline cooled at $0^{\circ} \mathrm{C}$. The wave that was eliminated by cortical cooling was considered to be cortical in origin.
In some of the experiments, monopolar recording of the LGB potential (A4.0, L4.0, and V-1.0 to -1.5) ipsilateral to the site of stimulation as referred to frontal sinus $(7 \mathrm{~mm}$ anterior to the bregma) was conducted in that an Elgiloy wire (Rocky Mountain/ Orthdontics, E273) electrode (250 $\mu \mathrm{m}$ in diam.) insulated except for a cross section of the electropolished tip of 20 to $30 \mu \mathrm{m}$ in diameter was used. The letter $\mathbf{V}$ represents the dorsoventral coordinate according to the atlas above mentioned.

Also, in some other experiments, SC or the lateral posterior nucleus of the thalamus (LPT) was unilaterally destroyed using a stainless steel monopolar anodal electrode $(200 \mu \mathrm{m}$ in diameter) insulated except for a 1-mm tip in the case of the SC lesion and a cross section of the electrode in the case of the LPT lesion. The lesion was made on the side on which CEP was recorded. The SC lesion was made at the site of A2.0, L1.6, and V0.5 by applying direct current as follows: in $0-10 \mathrm{sec}$, increased to $4 \mathrm{~mA}$; for 10 $30 \mathrm{sec}$, maintained at $4 \mathrm{~mA}$; in 30-35 sec, decreased to $0 \mathrm{~mA}$. The LPT lesion was made at the following sites: (1) A4.0, L2.8, V0.0; (2) A4.2, L2.8, V-0.2; (3) A4.4, L2.8, V0.0, at which direct current of $1 \mathrm{~mA}$ was passed for $10 \mathrm{sec}$.

A monopolar rectangular pulse of $0.1 \mathrm{msec}$ was used for stimulation of the left optic nerve. Thirty single potentials evoked by an electric shock with a frequency of $1.3 \mathrm{sec}$ were averaged by an ATAC-350 (Nihonkoden) with 1,024 data points at a sampling time of $50 \mu \mathrm{sec}$, and the results were recorded on graph paper.

Following completion of the recording, the rats were sacrificed with an overdose of pentobarbital. The brain with either the SC or LPT lesion was frozen-sectioned at $30 \mu \mathrm{m}$, and the size and location of the lesion were determined under a microscope. Severance of the optic tract was checked by visually inspecting several saggital sections of the brain (Figure 1).

\section{Recording Conditions and Analyses of CEP}

In intact adult rats, CEPs were recorded in the visual cortex either contralateral or ipsilateral to the side of the left optic nerve stimulation in order to know their general characteristics. For this purpose, 15 rats of 3 to 4 months of age were used. In addition, 6 more such rats were used to investigate the effect of the SC or LPT lesion upon CEPs. In these rats, the right eye was removed right after the head was fixed in the stereotaxic apparatus. Hereafter, these animals are called control rats.

Because it had been found that the LGB potential recorded ipsilateral to the remaining eye of rats with one eye removed at birth showed the most clear effect of enucleation (Sakai \& Yagi, 1981), the study concentrated on the analyses of CEP in rats that had had the right eye removed at birth and that had been allowed to survive for more than 3 months (OEB). In total, 19 OEBs served for the mapping of CEPs over the visual cortex and for the analyses of the lesion effect. It should be noted here that throughout the text "contralateral CEP" and "ipsilateral CEP" are used to refer to the evoked potentials recorded contralateral and ipsilateral to the side of optic nerve stimulation, respectively.

The amplitude of a wave of CEP was measured in vertical length from a rising point of the wave toward negativity to the negative peak, as illustrated in Figure 2C. The polarity of CEP is described in this paper as being based on the outer electrode (cortical surface) referred to the inner electrode (inside the cortex). The latency of the wave was defined as the time that had elapsed from the onset of stimulation to the rising point of the wave toward negativity.

\section{RESULTS}

Since, in each recording condition, the pattern of CEP and the distribution over the visual cortex were quite similar among all the subjects investigated, the following descriptions of CEP are based on one animal per condition. 

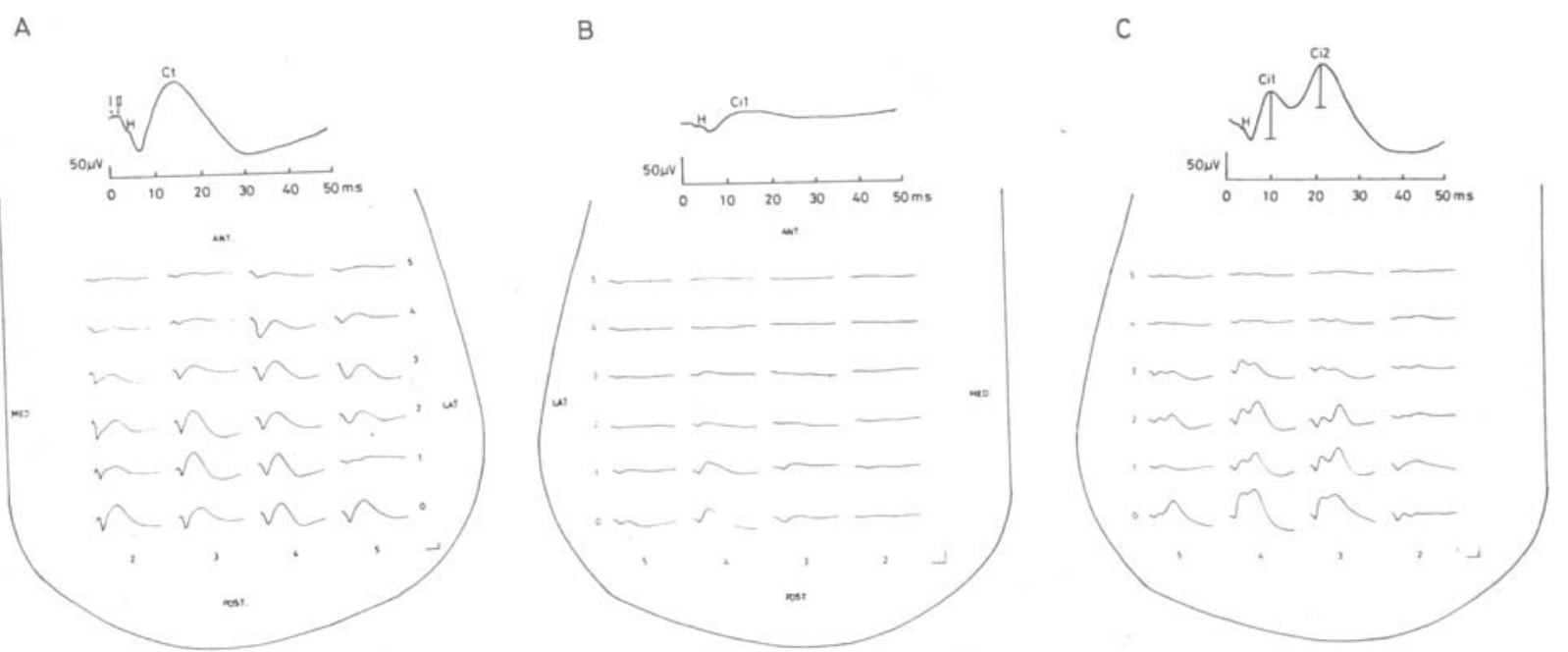

Figure 2. Dorsal views of right (A) and left (B and C) posterior cortices with CEPs contralateral (A) and ipsilateral (B and C) to the site of optic nerve stimulation. $A$ and $B$ are taken from two control rats, one for each hemisphere, and $C$ from one $O E B$ rat. CEPs recorded at $\mathrm{A1}$ and $\mathrm{L3}$ are magnified above. Scale: $50 \mu \mathrm{V}$ and $10 \mathrm{msec}$. In this and the following figures, upward deflections of CEPs indicate negativity of the electrode located at the cortical surface as referred to the inside of cortex.

\section{Contralateral and Ipsilateral CEPs in Control Rats}

Contralateral CEP. Ten rats were used. Figure 2A shows the typical contralateral CEP and the distribution over the visual cortex in one control rat when the optic nerve was stimulated at $25 \mathrm{~V}$. It was composed of two small waves (I and II) followed by one large wave $(\mathrm{Ct})$ with a small negative hump $(\mathrm{H})$ on a large surface positive (downward) deflection. Among the four waves, $\mathrm{Ct}$ was the only one that was definitely cortical in origin because it was completely eliminated by the cortical cooling. The threshold intensity for $\mathrm{Ct}$ to appear was 4 to $6 \mathrm{~V}$, at which the latency was around $5 \mathrm{msec}$. With an increase in stimulus intensity, the amplitude of $\mathrm{Ct}$ became larger, and when it was three to four times the threshold, the amplitude reached the maximum. However, the latency remained unaffected by this change in stimulus intensity.

As regards the distribution, the contralateral CEP was recorded from almost all the recording sites explored. The potential with the highest amplitude of Ct was usually obtained from recording sites located at $\mathrm{A} 0$ to $\mathrm{A} 2$ and $\mathrm{L} 3$ to L4.

Ipsilateral CEP. Five subjects were employed. Figure $2 \mathrm{~B}$ shows the typical ipsilateral CEP obtained by stimulation of the optic nerve at $25 \mathrm{~V}$ and the distribution in one control rat. The ipsilateral CEP has a slightly simpler configuration. It was composed of one relatively small wave (Ci1) with a tiny negative hump $(\mathrm{H})$ on a surface positive (downward) deflection. Only the Cil wave was found to be cortical in origin. The threshold intensity for Cil was about the same as that for $\mathrm{Ct}$, and so was the latency. An in- crease in intensity of stimulation did not affect the latency of $\mathrm{Cil}$, as in the case of $\mathrm{Ct}$, but it did affect the amplitude of Cil and to a lesser extent than in the case of Ct. By increasing the intensity of stimulation by only 2 to $3 \mathrm{~V}$ above threshold, the amplitude of Cil had already reached its maximum.

In contrast to the wide distribution of the contralateral CEP, the area from which the ipsilateral CEP could be recorded was very limited. It was recorded only from the area enclosed by A0 to A3 and L2 to L4.

\section{Contralateral and Ipsilateral CEPs \\ in the OEB Rat}

Contralateral CEP. Five OEB rats were investigated. The form and distribution of the contralateral CEP in the OEB were not different from those in the control rats and they are, therefore, not presented here.

Ipsilateral CEP. Eight OEB rats were used. Figure $2 \mathrm{C}$ illustrates the typical ipsilateral CEP and the distribution in one OEB rat when the optic nerve was stimulated at $24 \mathrm{~V}$. It was composed of two waves (Ci1 and $\mathrm{Ci2}$ ) with a small negative hump $(\mathrm{H})$ on the first surface positive (downward) deflection. Among these three waves, $\mathrm{Ci} 1$ and $\mathrm{Ci} 2$ were wiped out by cortical cooling. It was found that these two cortical waves were differently affected by the level of stimulus intensity. With weak stimulation, only Cil appeared. The threshold for evoking Cil and the stimulus strength that gave rise to the maximum Cil amplitude were almost the same as those for Cil in the control rat, and so were their latencies. However, there was one difference noted between the OEB rat 
and control rats, the amplitude being much larger in the OEB rat than in the control rat. For example, in the potential obtained at A1 and L3, the mean maximum amplitude of Cil was $84.5 \mu \mathrm{V}$ for the OEB rat $(\mathrm{N}=8)$ and $15.3 \mu \mathrm{V}$ for the control rat $(\mathrm{N}=5)$. The difference between the two means was significant $[\mathrm{t}=4.64, \mathrm{p}<.001]$.

When the intensity of stimulation was increased to about two or three times the threshold for Ci1, a new wave (Ci2) started to follow Ci1. The latency of $\mathrm{Ci} 2$ was around $18.0 \mathrm{msec}$. It should be noted that $\mathrm{Ci} 2$ was never recorded in the control rat. With a gradual increase in the intensity of stimulation, $\mathrm{Ci} 2$ increased in amplitude and the latency became shorter. And, when the stimulus intensity was about four or five times the threshold for $\mathrm{Ci} 1, \mathrm{Ci} 2$ reached its maximum in amplitude and its latency became the shortestaround 14.0 to $16.0 \mathrm{msec}$.

As shown in Figure 2C, the ipsilateral CEP was recorded from a much wider area of cortex in the OEB rat than in the control rats. The potential with a large amplitude of $\mathrm{Ci} 1$ and $\mathrm{Ci} 2$ was usually confined to the lateroposterior portion of the visual cortex, where the ipsilateral CEP could be obtained in the control rat. Thus, the overall distribution pattern of the ipsilateral CEP in the OEB rat resembled that of the contralateral CEP in the control rat (Figure 2A), although the amplitude of $\mathrm{Ct}$ was far greater than that of either Ci1 or Ci2. The mean Ct amplitude of control rats $(\mathrm{N}=10)$ at $\mathrm{A} 1$ and $\mathrm{L} 3$ was $235.0 \mu \mathrm{V}$, which was significantly larger than that of Ci1 noted earlier $[\mathrm{t}(16)=4.46, \mathrm{p}<.001]$ and that of $\mathrm{Ci} 2$ [mean amplitude, $47.9 \mu \mathrm{V} ; \mathrm{t}(16)=7.62, \mathrm{p}<.001]$ in the OEB rats $(\mathrm{N}=8)$.

\section{Extent of Lesions}

In the following two sections, the effects of SC and LPT lesions upon CEPs are described. Hence, it would be appropriate to summarize the anatomical results beforehand. The SC lesion was attempted in three OEB and three control rats. In all these rats, SC was destroyed from the stratum superficiale to the stratum album profundum. Laterally, whole portions of SC except for the medial $25 \%$ to $35 \%$ were damaged in two OEB and three control rats, and in one OEB the middle $60 \%$ to $75 \%$ of SC was damaged with some sparing tissues at both sides. The LPT lesion was attempted also in three OEB and three control rats. In two OEB rats and one control rat, damage was almost confined to LPT itself; in one OEB and one control rat it extended to parts of the pretectal nucleus, the ventral nucleus of the thalamus, and the posterior nucleus of the thalamus; in one control rat, it extended to parts of medial side of the LGB. However, since the LPT lesion alone or with damage of other brain structures affected CEPs in the same manner, the effect was considered mostly due to the LPT lesion.

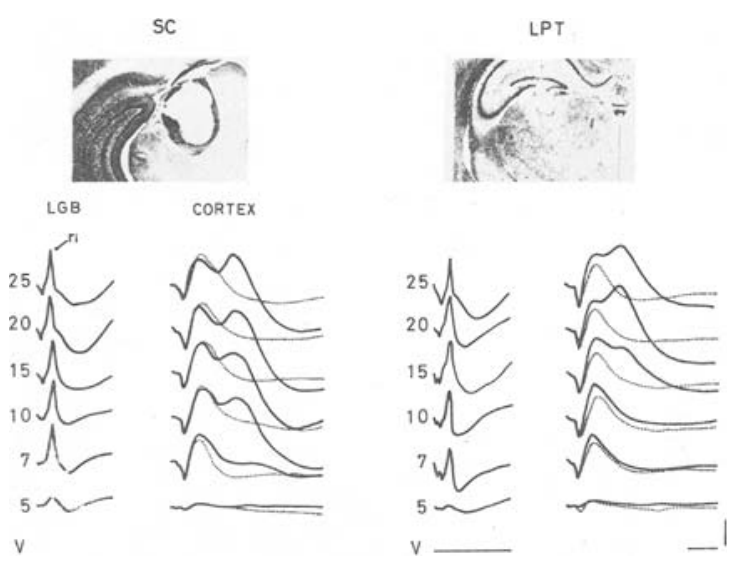

Figure 3. Ipsilateral LGB and cortical potentials evoked by stimulation of the optic nerve with six different intensities, and effect of SC lesion (left) and LPT lesion (right) upon CEPs in two OEB rats, one with each lesion. CEPs before lesioning are shown by solid lines and those after lesioning by dotted lines. The photographs above show parts of the actual lesions. Scale: $50 \mu \mathrm{V}$ and 10 msec.

\section{Projection Mediating $\mathrm{Ci} 1$ and $\mathrm{Ci} 2$}

An analysis was made to identify the projection that mediates $\mathrm{Ci} 1$ and $\mathrm{Ci} 2$ in the visual cortex in six OEB rats. Figure 3 illustrates the ipsilateral LGB potentials and CEPs in two OEB rats as the intensity of stimulation was increased stepwise from the threshold for Ci1. At every stimulus strength, the potential was first recorded from the LGB and then from the cortex. It was found that correlation of the amplitude of the LGB postsynaptic component (ri) with that of Ci1 was very high $(\mathrm{r}=.99-.96, \mathrm{~N}=6)$, whereas that with the amplitude of $\mathrm{Ci} 2$ was not so high $(\mathrm{r}=.57-.45, \mathrm{~N}=6)$. Judging from the results, Cil was believed to be mediated by the geniculostriate projection, but $\mathrm{Ci} 2$ was not. $\mathrm{Ci} 2$ was entirely eliminated by either the lesion of SC or LPT ipsilateral to the side of stimulation (Figure 3). Elimination of Ci2 was confirmed in all six rats, that is, by the SC lesion $(\mathrm{N}=3)$ and by the LPT lesion $(\mathrm{N}=3)$. The lesions did not change the latency of $\mathrm{Cil}$ and did not consistently affect the amplitude in a specific direction. Hence, $\mathrm{Ci} 2$ was assumed to be mediated mostly, if not exclusively, by the SC-LPT projection.

\section{The Effects of SC and LPT Lesions upon CEPs in Control Rats}

The effect of SC or LPT lesion was investigated on $\mathrm{Ct}$ in six control rats, three for each lesion. Figure 4 illustrates sample records. As seen in Figure 4, a comparison of $\mathrm{Ct}$ before and after the SC or LPT lesion suggests some contribution to potential $\mathrm{Ct}$ that was mediated by the SC-LPT projection. The effect of the SC or LPT lesion on Ct was noted in the shortening of time until the negative peak appeared after stimulation. It appeared around 15.0 to 
CONT-SC

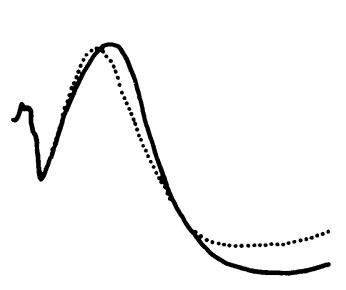

I

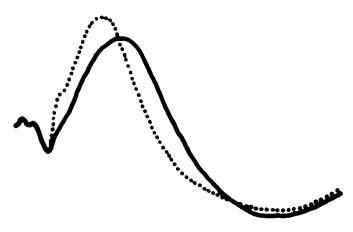

Figure 4. Effects of SC lesion (CONT-SC) and of LPT lesion (CONT-LPT) upon the contralateral CEPs in two control rats, one for each lesion. Potentials before lesions are shown by solid lines and those after lesions by dotted lines. Scale: $50 \mu \mathrm{V}$ and $10 \mathrm{msec}$.

$17.5 \mathrm{msec}$ before the lesion and around $13.0 \mathrm{msec}$ after the lesion. However, no consistent effect of the lesion was observed in the amplitude: increases, decreases, or no changes in the amplitude were observed in different control rats.

\section{DISCUSSION}

The present study clearly demonstrates that if one eye of a rat is enucleated at birth, a marked change in processing the uncrossed retinal input occurs in the visual cortex. As seen in Figure 2B, control ipsilateral potential has only one cortical wave (Ci1), and it is recorded from a limited lateroposterior portion of the visual cortex. The observation is in agreement with Montero's (1973) results. In contrast, the ipsilateral CEP of the OEB rat has not only $\mathrm{Ci1}$ but also a new wave, $\mathrm{Ci2}$, which is characteristic of the rat of this enucleation condition (Figure $2 \mathrm{C}$ ). $\mathrm{Ci} 1$ and $\mathrm{Ci} 2$ were greatly potentiated in amplitude in the OEB rat. They were both recorded in a wide area of the visual cortex. A marked potentiation of $\mathrm{Ci} 1$ and $\mathrm{Ci} 2$ was found in the area where the potential of Cil was most clearly obtained in the control rat, and potentiation became less clear as the recording site moved further away from that area in the anterior and medial directions. It is of interest to note the manner of the amplitude change of these two waves in OEB rats, which is, in a sense, quite similar to that of the enhanced postsynaptic component of the ipsilateral LGB potential previously reported (Sakai \& Yagi, 1981).

As for the projections mediating $\mathrm{Ci1}$ and $\mathrm{Ci} 2$, the present study suggests that Cil was mediated by a projection from LGB and $\mathrm{Ci} 2$ by a projection from SC-LPT. The interpretation is based mainly on the lesion effects of SC or LPT upon Ci1 and Ci2. However, one might argue against such an interpretation and suggest that the lesion effects might be due not to the lesion of SC or LPT, but rather to local cortical damage or damage to the geniculocortical axons made by the lesion electrode when it was lowered through the very cortex where the CEPs were recorded. However, if this is the case, it is difficult to explain why only $\mathrm{Ci} 2$ was selectively abolished and Cil was left unaffected by the lesion. Therefore, it seems unlikely that the lesion effects on $\mathrm{Ci} 1$ and $\mathrm{Ci} 2$ are due to the damage suggested by the above criticism.

A question arises, then, as to what structural and/ or functional base would underlie the enhancement and wide distribution of $\mathrm{Cil}$ and also the appearance of $\mathrm{Ci} 2$ in the visual cortex of the OEB rat.

As for the Cil, it should be taken into consideration that when monocular enucleation is performed within 30 postnatal days, stimulation of the remaining optic nerve evokes a potential in the ipsilateral LGB whose postsynaptic component is larger and more widely distributed than it is in normal rats (Sakai \& Yagi, 1981). The structural base for this LGB potential change is considered to be the expansion of the uncrossed retinogeniculate projection in LGB that is brought about by monocular enucleation at birth or in the early postnatal days (Lund et al., 1973), although the mechanism underlying this expansion is not so simple (Cunningham, 1976; Hickey, 1975; Seneglaub \& Finlay, 1981). This change in the LGB potential is obviously reflected by the potentiation of Cil and its wide distribution in the visual cortex. However, there is no study available to show that monocular enucleation at birth or in the early postnatal days of the rodent could produce structural and/or functional change in the terminals of the geniculocortical axons and the neuronal circuitry in the visual cortex, and, unfortunately, the present analysis of Cil can not provide any information on this problem. Hence, it remains to be seen if any functional reorganization in the structures mentioned above get involved in producing a final pattern of the Cil amplitude and distribution in the visual cortex.

What mechanism, then, could be responsible for the appearance of $\mathrm{Ci2}$ ? The present study suggests that it is mediated by SC-LPT projection. It has been histologically demonstrated in normal rats that LPT receives afferents from SC (Perry, 1980) and sends many efferents to the peristriate area and few to the striate cortex (Herkenham, 1980; Hughes, 1977; Perry, 1980). According to Krieg's (1946) atlas, major portions of the recording sites in the present study are believed to be located within the striate cortex. Therefore, it should be expected that the potential mediated by the LPT projection in the contralateral CEP would be very small if recorded. The present study confirmed this; no clear-cut potential related to that projection was obtained, and the lesion of LPT only made the time for the negative peak of $\mathrm{Ct}$ to appear slightly shorter (Figure 4). As mentioned earlier, monocular enucleation in the rat and hamster in the early postnatal days is known to pro- 
duce expanded retinotectal projection that is retinotopically organized and whose axons are ordered in a way that is appropriate for the normal contralateral target in the rat (Cunningham \& Speas, 1975). However, it should be stressed that the number of cells activated by the uncrossed optic fibers is still small compared with that activated by the crossed ones in the hamster (Rhoades, 1980). Therefore, it seems unlikely that the amount of input from SC to LPT mediated by the expanded uncrossed retinotectal fibers becomes greater than that mediated by the normal crossed ones. These considerations lead to the following prediction: recording of potential mediated by the expanded uncrossed retinotectal fiber-SC-LPT in the ipsilateral CEP of the OEB rat would be almost impossible, because no clear-cut potential related to the crossed retinotectal fiber-SCLPT was recorded even in the contralateral CEP in the control rat. However, the result was quite contrary to this prediction. Ci2 was clearly recorded in the OEB rat. How could this phenomenon be explained?

Recently, the accumulated evidence has indicated that, in the central nervous system, when a cell is deprived of its afferent, nearby axons can sprout to make effective contact with that cell (Lynch, Gall, Rose, \& Cotman, 1976; Raisman, 1969; Wall \& Egger, 1971). It is speculated that the same type of neuronal reorganization could have taken place in the ipsilateral visual cortex in the OEB rat. In this connection, Valverde's (1968) observation and speculation seem worthy of consideration. Using the mouse with one eye removed at birth, Valverde observed that the dendrites of many stellate cells in layer IV of the striate cortex ipsilateral to the remaining eye showed a tendency to shift towards layers III, II, and V because of the absence of the LGB input. He speculated that these dendrites would make synaptic contact with the recurrent collaterals of axons of superficial pyramidal cells and the cortical associational fibers as well as with the horizontal collaterals of axons of deep pyramidal cells. While the above speculation might be true, we think it equally probable that some of those shifted dendrites of stellate cells would also make effective contact with the proliferated terminals of LPT projection to the striate and peristriate cortices, and that this kind of reorganization would underlie the appearance of $\mathrm{Ci} 2$ in the OEB rat. An exact understanding of neuronal reorganization underlying this phenomenon must, of course, await further electrophysiological and histochemical analyses, such as those that are now under way in this laboratory.
Cunningham, T. J., \& Speas, G. Inversion of anomalous uncrossed projections along the mediolateral axis of the superior colliculus: Implications for retinocollicular specificity. Brain Research, 1975, 88, 73-79.

Finlay, B., Wilson, K. G., \& Schneider, G. E. Anomalous ipsilateral retinotectal projections in Syrian hamsters with early lesion: Topography and functional capacity. Journal of Comparative Neurology, 1979, 183, 721-740.

Frost, D. O., \& Schneider, G. E. Plasticity of retinofugal projections after partial lesions of the retina in newborn Syrian hamsters. Journal of Comparative Neurology, 1979, 185, 517-568.

Herkenham, M. Laminar organization of thalamic projections to the rat neocortex. Science, 1980, 207, 532-535.

HiCKEY, T. L. Translaminar growth of axons in the kitten dorsal lateral geniculate nucleus following removal of one eye. Journal of Comparative Neurology, 1975, 161, 359-382.

Hughes, H. Anatomical and neurobehavioral investigations concerning the thalamo-cortical organization of the rat's visual system. Journal of Comparative Neurology, 1977, 175, 311-336.

König, J. F. R., \& KLIPPEL, R. A. The rat brain: A stereotaxic atlas of the forebrain and lower parts of the brain stem. Baltimore: Williams \& Wilkins, 1963.

KRIEG, W. J. S. Connections of the cerebral cortex 1. The albino rat. A. Topography of the cortical areas. Journal of Comparative Neurology, 1946, 84, 221-276.

Lund, R. D., Cunningham, T. J., \& Lund, J. S. Modified optic projections after unilateral eye removal in young rats. Brain, Behavior and Evolution, 1973, 8, 51-72.

LunD, R. D., \& LuND, J. S. Reorganization of the retinotectal pathway in rats after neonatal retinal lesions. Experimental Neurology, 1973, 140, 377-390.

Lynch, G., Gall, C., Rose, G., \& Cotman, C. Changes in the distribution of the dentate gyrus associational system following unilateral or bilateral enthorhinal lesions in the adult rat. Brain Research, 1976, 110, 57-71.

Montero, V. M. Evoked responses in the rat's visual cortex to contralateral, ipsilateral and restricted photic stimulation. Brain Research, 1973, 53, 192-196.

Perry, V. H. A tectocortical visual pathway in the rat. Neuroscience, 1980, 5, 915-927.

Raisman, G. Neuronal plasticity in the septal nuclei of the rat. Brain Research, 1969, 14, 25-48.

RhoAdes, R. W. Effects of neonatal enucleation on the functional reorganization of the superior colliculus in the golden hamster. Journal of Physiology, 1980, 301, 383-399.

SAKAI, M., YAGI, F. Evoked potential in the lateral geniculate body as modified by enucleation of one eye in the albino rat. Brain Research, 1981, 210, 91-102.

Seneglaub, D. R., \& Finlay, B. L. Early removal of one eye reduces normally occurring cell death in the remaining eye. Science, 1981, 213, 573-574.

Takeuchi, H., Fukuda, Y., Hara, Y., \& Hsiao, C.-F. Physiological properties of expanded ipsilateral retinocollicular projection in neonatally enucleated albino rats. Brain Research, 1982, 231, 191-196.

Thompson, I. D. Changes in the uncrossed retinotectal projections after removal of the other eye at birth. Nature, 1979, 279, 63-66.

VALVERDE, F. Structural changes in the area striata of the mouse after enucleation. Experimental Brain Research, 1968, 5, 274-292.

WALl, P. D., \& EgGER, D. Formation of new connexions in adult brains after partial deafferentation. Nature, 1971, 232, 542-544.

\section{REFERENCES}

Cunningham, T. J. Early eye removal produces excessive bilateral branching in the rat: Application of cobalt filling method. Science, 1976, 14, 857-859.
(Manuscript received September 22, 1982; revision accepted for publication March 28, 1983.) 